Article

\title{
Ursolic Acid-Enriched Herba Cynomorii Extract Induces Mitochondrial Uncoupling and Glutathione Redox Cycling Through Mitochondrial Reactive Oxygen Species Generation: Protection Against Menadione Cytotoxicity in H9c2 Cells
}

\author{
Jihang Chen, Hoi Shan Wong and Kam Ming Ko * \\ Division of Life Science, Hong Kong University of Science and Technology, Clear Water Bay, \\ Hong Kong SAR, Hong Kong, China \\ * Author to whom correspondence should be addressed; E-Mail: bcrko@ust.hk; \\ Tel.: +852-2358-7298.
}

Received: 2 December 2013; in revised form: 19 January 2014 / Accepted: 21 January 2014 / Published: 27 January 2014

\begin{abstract}
Herba Cynomorii (Cynomorium songaricum Rupr., Cynomoriaceae) is one of the most commonly used 'Yang-invigorating' tonic herbs in Traditional Chinese Medicine (TCM). An earlier study in our laboratory has demonstrated that HCY2, an ursolic acidenriched fraction derived from Herba Cynomorii, increased mitochondrial ATP generation capacity (ATP-GC) and induced mitochondrial uncoupling as well as a cellular glutathione response, thereby protecting against oxidant injury in H9c2 cells. In this study, we demonstrated that pre-incubation of $\mathrm{H} 9 \mathrm{c} 2$ cells with $\mathrm{HCY} 2$ increased mitochondrial reactive oxygen species (ROS) generation in these cells, which is likely an event secondary to the stimulation of the mitochondrial electron transport chain. The suppression of mitochondrial ROS by the antioxidant dimethylthiourea abrogated the HCY2-induced enhancement of mitochondrial uncoupling and glutathione reductase (GR)-mediated glutathione redox cycling, and also protected against menadione-induced cytotoxicity. Studies using specific inhibitors of uncoupling protein and GR suggested that the HCY2-induced mitochondrial uncoupling and glutathione redox cycling play a determining role in the cytoprotection against menadione-induced oxidant injury in H9c2 cells. Experimental evidence obtained thus far supports the causal role of HCY2-induced mitochondrial ROS production in eliciting mitochondrial uncoupling and glutathione antioxidant responses, which offer cytoprotection against oxidant injury in H9c2 cells.
\end{abstract}


Keywords: Cynomorium songaricum; ursolic acid; mitochondrial uncoupling; glutathione redox cycling; oxidant injury

\section{Introduction}

The mitochondrion serves as a platform for cellular energy metabolism as well as signal transduction pathways relevant to the regulation of cell survival and death [1]. Mitochondrial dysfunction is believed to be pathologically related to various forms of cardiovascular diseases such as heart failure [2]. The maintenance of mitochondrial functional integrity and antioxidant capacity is therefore critical for cell survival, particularly for cardiac cells under oxidative stress conditions. In this connection, 'Yang-invigoration' in Traditional Chinese Medicine (TCM), which is pharmacologically viewed as the enhancement of mitochondrial ATP generation and the associated increase in mitochondrial antioxidant capacity, may offer a promising prospect for preventing oxidant injury in the heart [3].

Herba Cynomorii (the fresh stem of Cynomorium songaricum Rupr., Cynomoriaceae; also known as Suo-Yang in Chinese) is one of the most commonly used 'Yang-invigorating' Chinese tonic herbs. This herb is mainly found distributed across the deserts in Western China, such as Xinjiang, Qinghai, Gansu, Ningxia, Inner Mongolia and Shanxi, as well as in Central Asia, Iran and Mongolia. In TCM practice Herba Cynomorii is prescribed for treating lumbar weakness and enhancing sexual ability for both men and women. In recent years, the major constituents of Herba Cynomorii have so far been identified as phenolic compounds, steroids, triterpenes, among other constituents [4]. In addition, pharmacological investigations have shown that certain fractions and chemical compounds isolated from Herba Cynomorii possess a wide spectrum of biological activities, including anti-HIV protease [5], anti-HCV protease [6], anti-apoptosis, antioxidant, anti-aging, anti-fatigue, anti-osteoporotic, anti-diabetic and fertility promoting actions [7,8]. A previous study in our laboratory has demonstrated that HCY2, an ursolic acid (UA) -enriched fraction derived from Herba Cynomorii increased mitochondrial ATP generation capacity (ATP-GC) and induced mitochondrial uncoupling as well as a cellular glutathione response, resulting in protection against oxidant injury in H9c2 cells [9]. However, the biochemical mechanism underlying the induction of these cellular protective responses by HCY2 is still unknown. With reference to other experimental findings on Yang-tonic herbs or formulas [10,11], we hypothesized that 'Yang-invigoration' induced by HCY2 may involve a sustained and low level of mitochondrial reactive oxygen species (ROS) production, which is secondary to the increased activity of the electron transport chain [12]. The increased formation of ROS within mitochondria would further trigger cellular responses, including mitochondrial uncoupling and glutathione redox cycling [13], with resultant protection against oxidant injury. To test this hypothesis, we examined the effect of HCY2 on mitochondrial ROS production and provided evidence for the supporting the role of mitochondrial ROS in HCY2-induced mitochondrial uncoupling and glutathione redox cycling in H9c2 cells. The effects of an antioxidant and specific inhibitors of uncoupling protein (UCP) and glutathione reductase (GR), an enzyme for catalyzing the regeneration of reduced glutathione (GSH) from oxidized glutathione (GSSG), on HCY2-induced protection against menadione-induced cytotoxicity were also investigated. 


\section{Results and Discussion}

The extent of eletron transport in isolated mitochondria was measured by monitoring the reduction of MTT. As shown in Figure 1, pre-incubation of H9c2 cells with HCY2 concentration-dependently increased the extent of mitochondrial electron transport supported by pyruvate and malate, with the extent of stimulation being $32 \%$ and $75 \%$ relative to control at 10 and $25 \mu \mathrm{g} / \mathrm{mL}$, respectively.

Figure 1. Effects of pre-incubation of H9c2 cells with HCY2 on mitochondrial electron transport. Cells were pre-incubated with HCY2 (10 or $25 \mu \mathrm{g} / \mathrm{mL})$ for $4 \mathrm{~h}$. Pyruvate and malate-supported mitochondrial electron transport was measured by MTT reduction, as described in the Experimental Section. Data are expressed as the percentage of non-herbal extract pre-incubated control values $(0.072 \pm 0.009)$. Each bar represents the mean $\pm \mathrm{SD}$, with $n=6$. * Significantly different from the control group without HCY2 pre-incubation $(p<0.05)$.

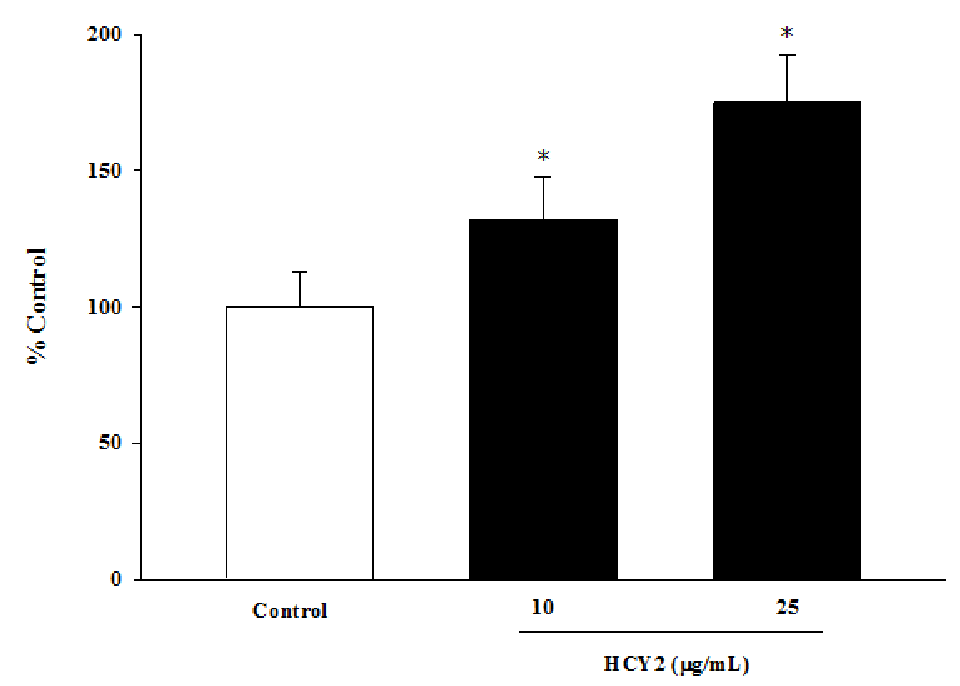

As shown in Figure 2, the HCY2 (10 or $25 \mu \mathrm{g} / \mathrm{mL})$-induced stimulation of mitochondrial electron transport was accompanied by a small but significant increase in mitochondrial ROS production (11\% and 23\% respectively). Although DMTU (a thiol-containing antioxidant; $20 \mathrm{mM}$ ) per se did not alter mitochondrial ROS production, co-incubation of HCY2 $(25 \mu \mathrm{g} / \mathrm{mL})$ with DMTU almost completely abrogated the HCY2-induced mitochondrial ROS production in $\mathrm{H} 9 \mathrm{c} 2$ cells.

To investigate whether the stimulation of mitochondrial electron transport is accompanied by increases in mitochondrial membrane potential, the changes in mitochondrial membrane potential were monitored. Figure 3a shows that incubation of $\mathrm{H} 9 \mathrm{c} 2$ cells with $\mathrm{HCY} 2(25 \mu \mathrm{g} / \mathrm{mL})$ caused time-dependent increases in mitochondrial membrane potential during the initial time period (0-10 min), with the maximum extent of stimulation being $16 \%$ at $10 \mathrm{~min}$. Interestingly, there were gradual decreases in mitochondrial membrane potential following the 10-min incubation with HCY2 $(25 \mu \mathrm{g} / \mathrm{mL})$, with the extent of decrease being $8 \%$ at $40 \mathrm{~min}$, when compared with the vehicle control. However, FCCP, a chemical uncoupler of mitochondria, was found to cause a gradual decrease in mitochondrial membrane potential, the extent of reduction being approximately $16 \%$ at the end of the 40 -min incubation. As shown in Figure $3 \mathrm{~b}$, guanosine diphosphate (GDP, a UCP inhibitor) did not affect the mitochondrial membrane 
potential when compared with the control group. The co-incubation of the H9c2 cells with GDP during the exposure to HCY2 was found to inhibit the decreases in mitochondrial membrane potential at both tested concentrations of HCY2. It should be noted that the co-incubation with GDP did not affect the FCCP-induced decreases in mitochondrial membrane potential.

Figure 2. Effects of HCY2 on mitochondrial ROS production in H9c2 cells. Cells were pre-incubated with HCY2 (10 or $25 \mu \mathrm{g} / \mathrm{mL})$ with or without DMTU $(20 \mathrm{mM})$ for $4 \mathrm{~h}$. Mitochondrial ROS production was measured as described in the Experimental Section. Values given are mean $\pm \mathrm{SD}$, with $n=3$. * Significantly different from the control group without HCY2 pre-incubation; \# significantly different from the HCY2-pre-incubated group $(p<0.05)$.

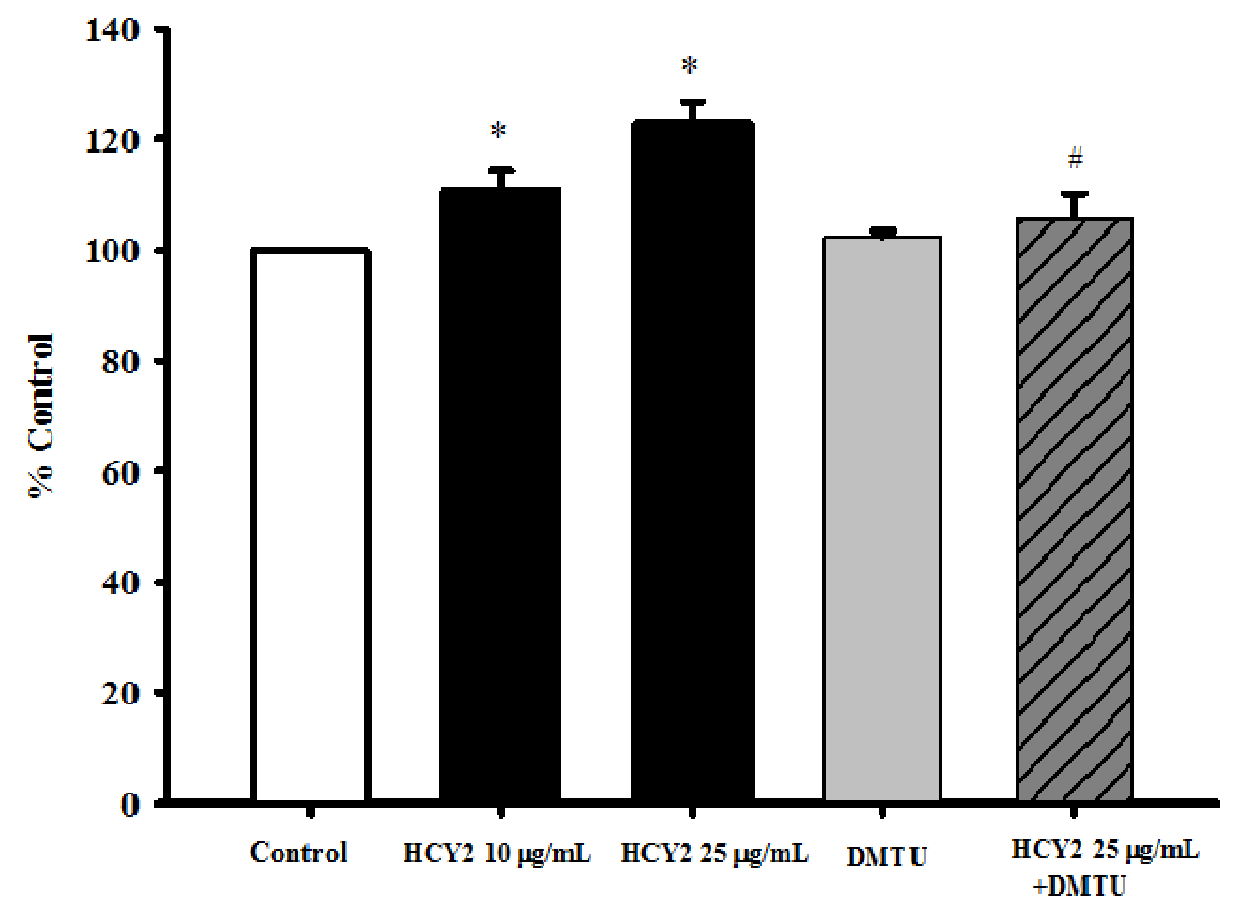

Table 1 shows that pre-incubation of $\mathrm{H} 9 \mathrm{c} 2$ cells with $\mathrm{HCY} 2$ at $25 \mu \mathrm{g} / \mathrm{mL}$ not only increased state 3 respiration (by $41 \%$ ), but also stimulated state 4 respiration (by $78 \%$ ). Consequently, the ratio of state 3 to state 4 respiration rate, which is also referred to as the 'respiration control ratio' (RCR, an index of mitochondrial coupling efficiency), was decreased (by 20\%). The uncoupling effect induced by HCY2 (25 $\mu \mathrm{g} / \mathrm{mL}$ ) was completely abrogated by GDP. To explore the role of ROS in HCY2-induced mitochondrial uncoupling, H9c2 cells were co-incubated with HCY2 $(25 \mu \mathrm{g} / \mathrm{mL})$ and DMTU $(20 \mathrm{mM})$, and mitochondrial respiration was measured. The result showed that DMTU per se did not alter mitochondrial respiration when compared with the control group. Although co-incubation with DMTU did not affect the HCY2-induced increase in state 3 respiration, the HCY2-induced stimulation of state 4 respiration was almost completely suppressed by DMTU. Consequently, DMTU co-incubation completely abrogated the HCY2-induced mitochondrial uncoupling, as indicated by the increased RCR value.

As shown in Figure 4, incubation of $\mathrm{H} 9 \mathrm{c} 2$ cells with $\mathrm{HCY} 2$ at $25 \mu \mathrm{g} / \mathrm{mL}$ produced time-driven cyclic variations in cellular GSH levels, with the increase in the amplitude of oscillation being $35 \%$, when compared with the control. While DMTU $(20 \mathrm{mM})$ did not produce any detectable changes in 
cellular GSH levels, BCNU $(50 \mu \mathrm{M})$ significantly decreased the cellular GSH levels when compared with the control group. The HCY2-induced time-driven cyclic variation of cellular GSH level, referred to as glutathione redox cycling, was largely abrogated by DMTU and BCNU.

Figure 3. Effects of HCY2 on mitochondrial membrane potential in H9c2 cells. Cells were incubated with HCY2 (10 or $25 \mu \mathrm{g} / \mathrm{mL}$ ) without (a) or with (b) GDP (500 $\mu \mathrm{M})$. FCCP $(100 \mu \mathrm{M})$, a chemical uncoupler, was used as a positive control in this experiment. Mitochondrial membrane potential was measured as described in the Experimental Section. Data are expressed as percent control with respective to the time-matched non-herbal extract pre-incubated control. Values given are mean $\pm \mathrm{SD}$, with $n=3$. * Significantly different from the control group without HCY2 pre-incubation $(p<0.05)$.

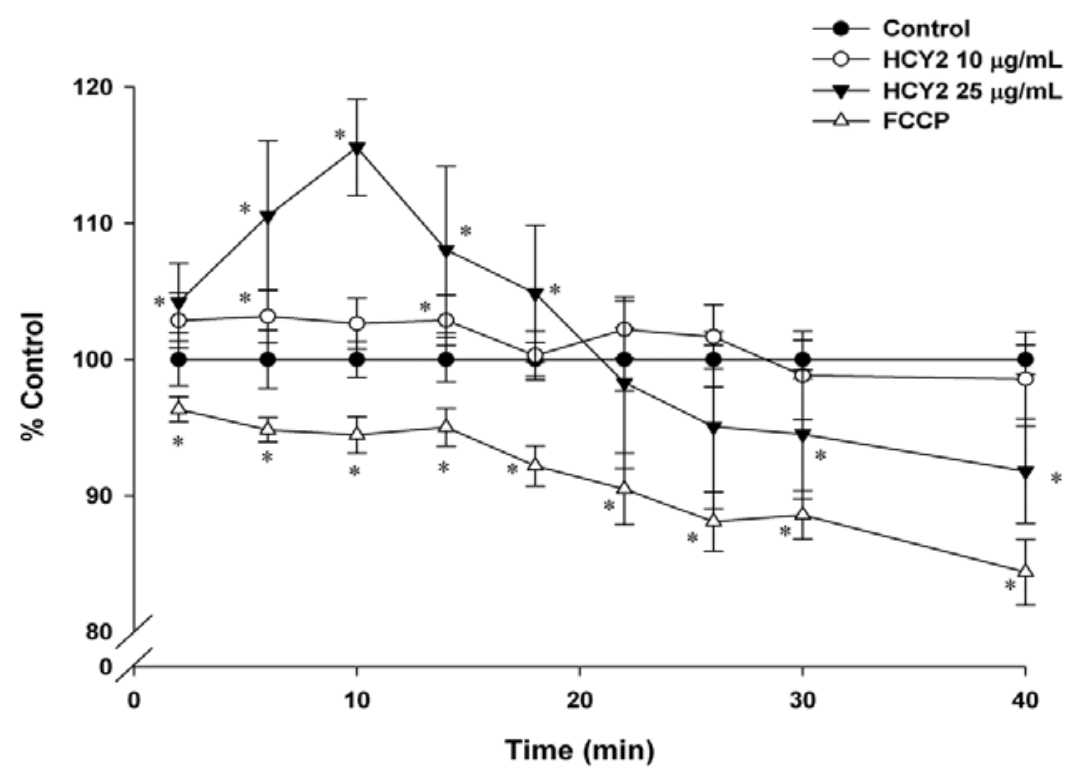

a
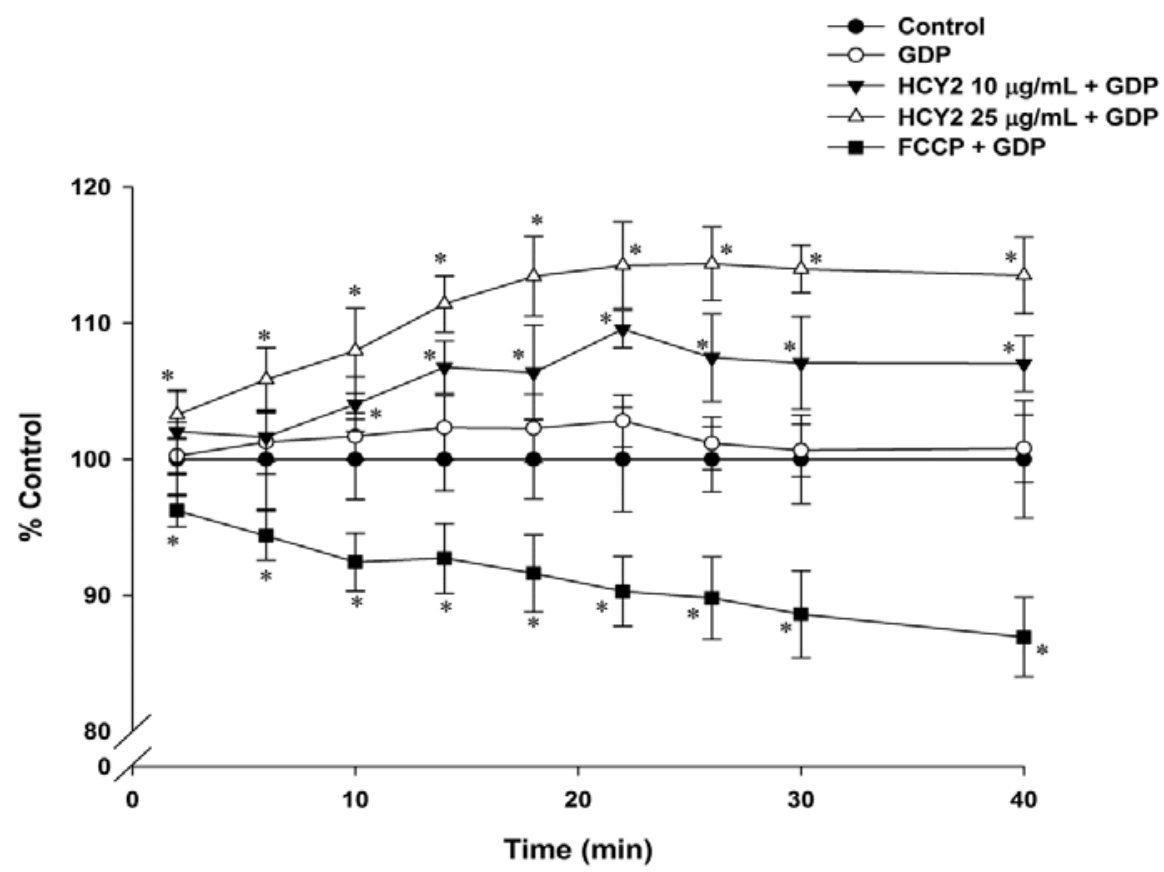
Table 1. Effects of HCY2 on mitochondrial respiration in $\mathrm{H} 9 \mathrm{c} 2$ cells in the absence or presence of GDP or DMTU. * Significantly different from the control group without HCY2 pre-incubation; \# significantly different from the HCY2-pre-incubated group $(p<0.05)$

\begin{tabular}{ccccccc}
\hline Respiration rate & Control & HCY2 & GDP & HCY2 + GDP & DMTU & HCY2 + DMTU \\
\hline State 3 & $100.00 \pm 3.02$ & $145.70^{*} \pm 12.48$ & - & - & $104.27 \pm 5.68$ & $139.22^{*} \pm 3.86$ \\
State 4 & $100.00 \pm 3.77$ & $177.80^{*} \pm 13.22$ & $98.68 \pm 2.47$ & $95.45^{\#} \pm 3.27$ & $102.04 \pm 5.93$ & $107.64^{\#} \pm 4.88$ \\
State 3/State 4 & $100.00 \pm 1.88$ & $81.83^{*} \pm 2.57$ & $101.23 \pm 4.31$ & $152.86^{* \#} \pm 13.64$ & $102.10 \pm 0.54$ & $129.73^{* \#} \pm 6.80$ \\
\hline
\end{tabular}

Figure 4. Effects of DMTU and BCNU on HCY2-induced enhancement on the glutathione redox cycling in $\mathrm{H} 9 \mathrm{c} 2$ cells. Cells were incubated with $\mathrm{HCY} 2(25 \mu \mathrm{g} / \mathrm{mL})$ with or without DMTU $(20 \mathrm{mM})$ or BCNU $(50 \mu \mathrm{M})$. Cellular GSH levels were measured as described in the Experimental Section. Data are expressed as the percentage of non-herbal extract or non-drug pre-incubated parallel control values (initial control GSH level $=25.01 \pm 1.96 \mathrm{nmol} / \mathrm{mg}$ protein) at various periods of herbal extract or drug incubation. Values given are means $\pm \mathrm{SD}$, with $n=3$. * Significantly different from the control group without HCY2 pre-incubation; \# significantly different from the HCY2-pre-incubated group $(p<0.05)$.

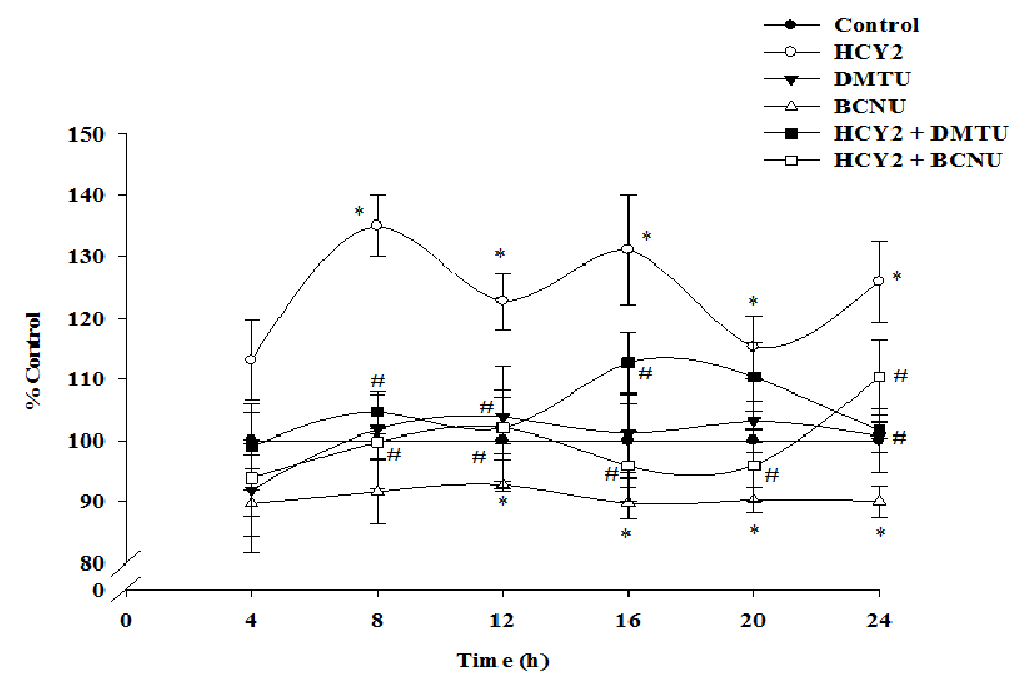

To determine the biological significance of the HCY2-induced cellular responses, the effect of HCY2 on menadione-induced oxidant injury in H9c2 cells was examined. As shown in Figure 5, HCY2 at both 10 and $25 \mu \mathrm{g} / \mathrm{mL}$ did not produce any detectable changes in LDH leakage in H9c2 cells. Menadione caused cellular injury in H9c2 cells, as evidenced by the significant increase in the extent of LDH leakage (148\%). H9c2 cells were pre-incubated with HCY2 (10 or $25 \mu \mathrm{g} / \mathrm{mL})$ and subjected to menadione challenge. The result showed that prior exposure to HCY2 significantly suppressed the menadione-induced LDH leakage in a concentration-dependent manner, with the extent of protection at the two tested concentrations of HCY2 being $22 \%$ and $42 \%$, respectively. As shown in Figure $6 a$, DMTU and GDP did not alter the LDH leakage under non-menadione and menadione challenge conditions. Although BCNU did not alter LDH release under non-menadione challenge conditions, it increased the sensitivity of H9c2-treated cells to menadione toxicity. When co-incubated with DMTU or BCNU, HCY2 did not suppress the menadione-induced LDH release as compared with the 
respective control. In other words, the cytoprotection afforded by HCY2 was completely abrogated by both DMTU and BCNU co-incubation. However, the use of GDP only partially suppressed the cytoprotective effect of HCY2 against menadione-induced cytotoxicity (Figure 6b).

Figure 5. Effects of HCY2 on menadione-induced cytotoxicity in $\mathrm{H} 9 \mathrm{c} 2$ cells. Cells were pre-incubated with HCY2 (10 or $25 \mu \mathrm{g} / \mathrm{mL}$ ) for $4 \mathrm{~h}$, followed by another $4 \mathrm{~h}$-incubation with or without menadione $(12.5 \mu \mathrm{M})$. The extent of LDH release was estimated as described in the Experimental Section. Values given are means $\pm \mathrm{SD}$, with $n=4$. * Significantly different from the non-menadione challenged control group $(p<0.05)$; \# significantly different from the menadione challenged control group $(p<0.05)$.

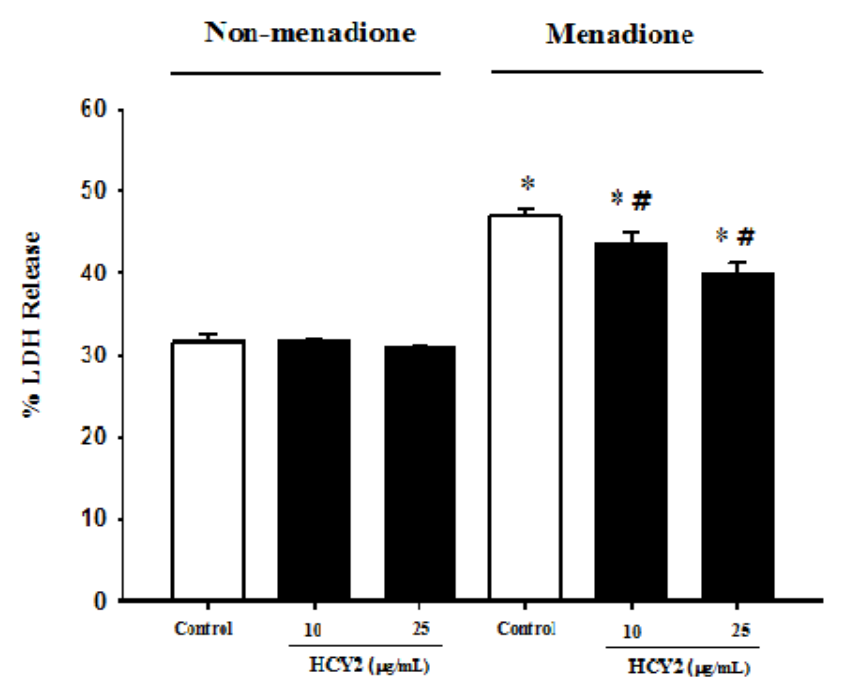

Figure 6. Effects of DMTU, BCNU and GDP on HCY2-induced protection against menadione toxicity in H9c2 cells. Cells were pre-incubated with HCY2 $(25 \mu \mathrm{g} / \mathrm{mL})$ in the absence or presence of DMTU $(20 \mathrm{mM})$, BCNU $(50 \mu \mathrm{M})$ or GDP $(500 \mu \mathrm{M})$ for $4 \mathrm{~h}$, followed by another $4 \mathrm{~h}$ incubation with menadione $(12.5 \mu \mathrm{M})$. (a) The percentage of lactate dehydrogenase (LDH) release was measured as described in the Experimental Section; and (b) The degree of protection against menadione-induced cytotoxicity was estimated as described in the Experimental Section.

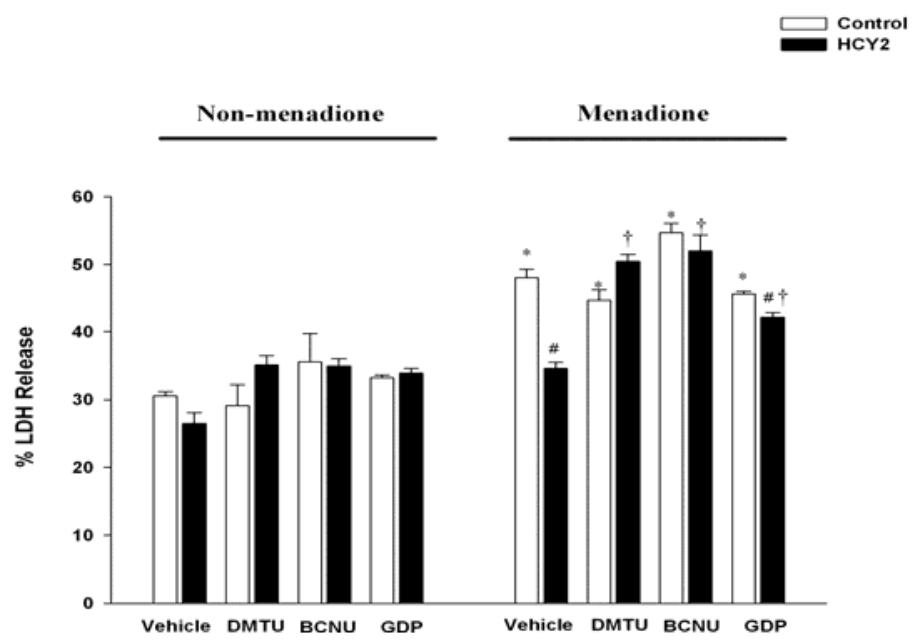

(a) 
Figure 6. Cont.

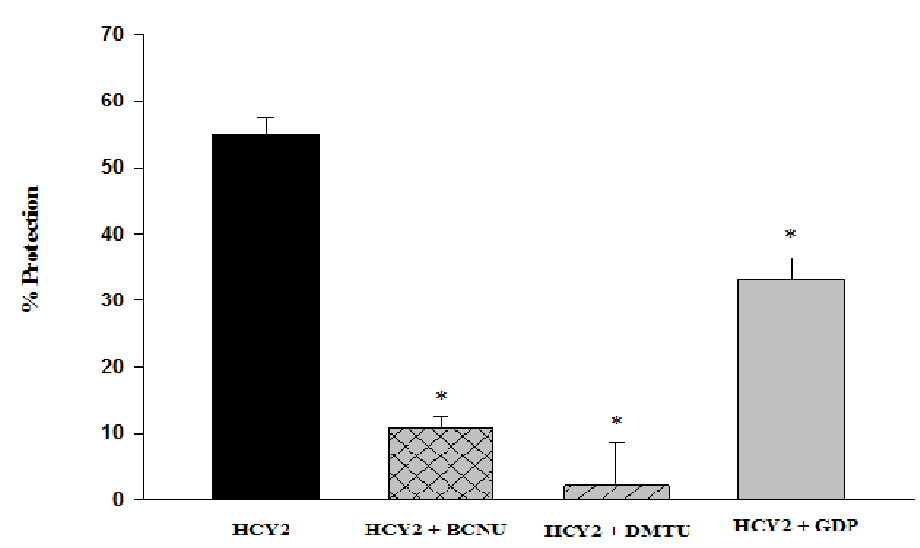

(b)

(a)* Significantly different from the respective non-menadione control group; \# significantly different from the respective manadione control group; $\dagger$ significantly different from the respective HCY2 pre-incubated menadione group $(p<0.05)$; $(\mathbf{b}) *$ Significantly different from the HCY2 pre-incubated group $(p<0.05)$. Values given are means $\pm \mathrm{SD}$, with $n=3$.

Figure 7 shows that while menadione-induced cytotoxicity was accompanied by a drastic decrease in cellular GSH levels (57\%), HCY2 pre-incubation at $25 \mu \mathrm{g} / \mathrm{mL}$ increased cellular GSH levels in $\mathrm{H} 9 \mathrm{c} 2$ cells under both non-menadione and menadione-challenged conditions. While BCNU decreased GSH levels under both non-menadione and menadione-challenged conditions, DMTU did not alter GSH levels when compared with their respective controls. The HCY2-induced increase in GSH levels was completely suppressed by the c o-incubation with BCNU and DMTU when compared with the HCY2 group.

Mitochondria not only serve as the powerhouse of the cell, but they also are the primary source of cellular ROS production [14]. The electron transport chain, in which a number of one-electron transfer reactions occur, has been recognized as the most important site of ROS generation in mitochondria [15]. Although ROS are usually considered as culprits in the pathogenesis of a number of diseases, a compelling body of evidence has demonstrated that low levels of ROS production also play an important role in intracellular redox homeostasis and signal transduction [16].

A previous study in our laboratory has demonstrated the ability of an ethanol extract of Herba Cynomorii to increase mitochondrial ATP-GC, which was associated with the stimulation of pyruvatesupported mitochondrial electron transport in H9c2 cells [10]. In the present study, HCY2, a UA-enriched fraction isolated from an ethanol extract of Herba Cynomorii, was found to stimulate mitochondrial electron transport in situ in H9c2 cells. As mitochondrial ROS are unavoidably produced as a consequence of the oxidative phosphorylation process, particularly under conditions of increased electron transport activity [17], the stimulation of mitochondrial electron transport by HCY2 was associated with an increased mitochondrial ROS production. The increase in mitochondrial electron transport was also accompanied by an increase in mitochondrial membrane potential, with the latter inhibiting further electron transport but favoring ROS production [17]. Superoxide anion radicals generated during mitochondrial respiration are capable of activating UCPs on the inner mitochondrial membrane, thereby lowering the membrane potential through dissipation of the proton gradient which is its major determinant [18]. With respect to uncoupled respiration, our preliminary study indicated 
that HCY2 increased the expression of UCP3 in cultured $\mathrm{C} 2 \mathrm{C} 12$ cells (unpublished data). The uncoupling effect of UCPs could therefore be inhibited by purine nucleotide diphosphate, such as GDP [18]. Under the present experimental conditions, GDP, was found to reverse the decrease in mitochondrial membrane potential induced by HCY2, suggesting the involvement of activation of mitochondrial UCPs in the process. The possible involvement of UCPs in HCY2-induced uncoupled respiration is supported by the finding that GDP can reverse the HCY2-induced decrease in RCR, as observed in the present study [19]. Moreover, the UCP-mediated-mitochondrial uncoupling induced by HCY2 was completely prevented by the co-incubation with DMTU, suggesting that the uncoupling effect of HCY2 may also be related to ROS production.

Figure 7. Effects of $\mathrm{HCY} 2$ on cellular GSH levels in non-menadione and menadione-challenged $\mathrm{H} 9 \mathrm{c} 2$ cells in the absence or presence of DMTU or BCNU. Cells were pre-incubated with HCY2 $(25 \mu \mathrm{g} / \mathrm{mL})$ in the absence or presence of DMTU $(20 \mathrm{mM})$ or BCNU $(50 \mu \mathrm{M})$ for $4 \mathrm{~h}$, followed by another $4 \mathrm{~h}$-incubation with or without menadione $(12.5 \mu \mathrm{M})$. Cellular GSH levels were measured under non-menadione or menadione challenged conditions as described in the Experimental Section. Data are expressed as the percentage of non-menadione challenged control value $(23.45 \pm 0.16 \mathrm{nmol} / \mathrm{mg}$ protein). Values given are means $\pm \mathrm{SD}$, with $n=3$. * Significantly different from the non-menadione challenged control group $(p<0.05)$; \# significantly different from the menadione challenged control group $(p<0.05)$; $\uparrow$ significantly different from the respective HCY2-pre-incubated group $(p<0.05)$

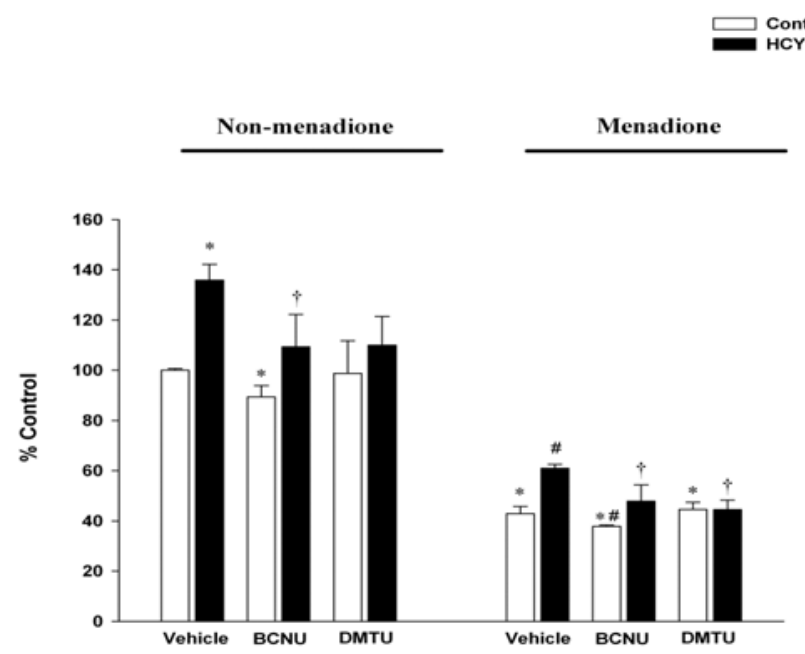

GSH is regarded as the first line of defense in preventing cellular oxidative damage [20]. In the present study, incubation of $\mathrm{H} 9 \mathrm{c} 2$ cells with $\mathrm{HCY} 2$ was found to induce a time-dependent cyclic variation in cellular GSH levels, indicative of an up-regulation of glutathione redox cycling. BCNU was used to investigate the role of GR in the glutathione response. The finding of GSH depletion following incubation with BCNU supports the involvement of GSH regeneration in the maintenance of cellular GSH status in $\mathrm{H} 9 \mathrm{c} 2$ cells. BCNU co-incubation abrogated the HCY2-induced cyclic changes in cellular GSH in H9c2 cells, suggesting the involvement of GSH regeneration from GSSG in the process. Although DMTU did not affect the cellular GSH level in H9c2 cells, the enhancement of glutathione redox cycling by HCY2 was also attenuated by the co-incubation with DMTU, implicating 
the involvement of mitochondrial ROS production in the HCY2-induced cellular antioxidant response. Conceivably, the prolonged incubation with HCY2 could lead to a continuous production of a small amount of signaling ROS in association with the increase in mitochondrial electron transport. The increased level of ROS would oxidize and hence deplete cellular GSH, with the resultant activation of GR-catalyzed regeneration of GSH from GSSG. Therefore, the activation of glutathione redox cycling is presumably related to the interplay between HCY2-induced ROS generation and the GR-catalyzed regeneration of GSH. Menadione, at high concentrations, is considered to be a cytotoxic substance that can undergo redox cycling through the catalysis of flavoprotein reductase, resulting in oxidative stress-induced tissue injury [21]. Our study demonstrated that incubation with HCY2 significantly protected against menadione-induced cytotoxicity in $\mathrm{H} 9 \mathrm{c} 2$ cells. To determine the role of mitochondrial ROS in protecting against menadione cytotoxicity, a thiol-containing antioxidant, DMTU, was used in this study. Although it would be expected that DMTU would protect against cytotoxicity by scavenging free radicals produced from menadione, the result showed that DMTU did not produce any detectable effect on menadione-induced LDH leakage or cellular GSH depletion in H9c2 cells, which was consistent with previous experimental data in our laboratory [22]. Indeed, Kossenjans et al. demonstrated that exposure to menadione at much higher concentrations $(100-200 \mu \mathrm{M})$ for $10 \mathrm{~h}$ induced drastic LDH leakage in bovine heart microvascular endothelial cells, which was preventable by DMTU [23]. By contrast, another research group reported that DMTU could not attenuate menadione $(15 \mu \mathrm{M}, 4 \mathrm{~h})$-induced LDH release and cellular GSH depletion in endothelial cells [24], in which the experimental conditions were similar to those in the present study. The differential effects of DMTU on menadione cytotoxicity may be due to the fact that prolonged exposure to high concentrations of menadione produced free radicals in larger amounts than that of a brief exposure to lower concentrations. By virtue of its free radical scavenging activities, DMTU could reduce the extent of cellular injury induced by high concentrations of menadione. However, DMTU did not attenuate cellular injury caused by low concentrations of menadione presumably because it was unable to reduce the extent of GSH depletion which is detrimental to cell survival.

The protection against oxidant injury afforded by HCY2 was completely abrogated by DMTU co-incubation, suggesting the involvement of ROS-mediated processes in the cytoprotective effect. The HCY2-induced increase in cellular GSH levels was also attenuated by DMTU under both control and menadione-intoxicated conditions. To define the role of GSH regeneration in the cytoprotection afforded by $\mathrm{HCY} 2$, the GR inhibitor (BCNU) was used as an experimental tool. The enhanced susceptibility of BCNU-pre-incubated cells to menadione cytotoxicity supports the role of GSH regeneration in maintaining cell survival under conditions of oxidative stress. BCNU was also found to decrease GSH levels following menadione exposure when compared with corresponding controls. The protective effect of HCY2 on menadione-induced toxicity was largely abolished by the co-incubation with the GR inhibitor. This is consistent with the observation that the decrease in the extent of protection against menadione toxicity afforded by HCY2 pre-incubation was paralleled by an attenuation in the HCY2-induced GSH antioxidant response by BCNU. The results therefore suggest that HCY2-induced glutathione redox cycling plays a determining role in protecting against menadione cytotoxicity in $\mathrm{H} 9 \mathrm{c} 2$ cells. In addition, GDP was used to delineate the role of mitochondrial uncoupling in the cytoprotection afforded by HCY2. Our results indicated that GDP did not affect menadione-induced LDH leakage. The co-incubation with GDP partially abolished the cytoprotection 
afforded by HCY2 against menadione challenge, implicating the involvement of mitochondrial uncoupling in this process.

Our preliminary study indicated that UA, the major component in HCY2, produced similar effects on ROS production and cytoprotection as the HCY2 preparation (unpublished data). UA has also been found to be a potential cardioprotective agent, which may act at least in part through the uncoupling of mitochondrial oxidative phosphorylation [25]. However, one cannot exclude the possibility that other constituents in HCY2 may also exert antioxidant effects that can protect against menadione cytotoxicity. In this regard, further comparative studies on mechanisms underlying the cytoprotective effect of UA and HCY2 on oxidant injury in H9c2 cells are warranted.

Taken collectively, our results indicate that the cytoprotection afforded by HCY2 against menadione toxicity is at least partly contributed to by UCP-mediated mitochondrial uncoupling, which is an event secondary to increased ROS production. More importantly, the elicitation of a glutathione antioxidant response, which is primarily caused by an increase in ROS production, plays a crucial role in the cytoprotective action of HCY2.

\section{Experimental}

\subsection{Chemicals}

Dulbecco's Modified Eagle's Medium (DMEM) and fetal bovine serum (FBS) were purchased from Gibco BRL Life Technologies (Grand Island, NY, USA). 2',7'- dichlorofluorescein diacetate (DCFDA) was purchased from Fluka (Buchs, Switzerland). Other chemicals were purchased from Sigma Chemical (St Louis, MO, USA). All chemicals were of analytical grade.

\subsection{Herbal Material and Extraction}

Herba Cynomorii was purchased from Lee Hoong Kee, Ltd., a local Hong Kong-based herbal dealer. The herb was authenticated by the supplier and a voucher specimen (HKUSTY01001) was deposited in the Division of Life Science, the Hong Kong University of Science and Technology (HKUST). An UA-enriched active fraction, HCY2, was isolated from an ethanol extract of Herba Cynomorii as previously described [9]. The content of UA in HCY2 was determined by using an HPLC-UV method. HPLC analysis was performed using an Agilent RRLC 1200 series system (Agilent, Waldbronn, Germany). HCY2 (dissolved in acetonitrile) was separated on a Waters Atlantis C18 column ( $5 \mu \mathrm{m}$ id, $4.6 \mathrm{~mm} \times 150 \mathrm{~mm}$ ). The mobile phase was composed of acetonitrile (solvent A) and $0.1 \%$ formic acid in water (solvent $\mathrm{B}$ ), using an isocratic gradient $78 \%$ (solvent $\mathrm{A}$ ) for $50 \mathrm{~min}$. The detection wavelength was set at $210 \mathrm{~nm}$. The quantitation of UA in the HCY2 fraction was determined from a calibration curve. A standard stock solution of UA $(5 \mathrm{mg} / \mathrm{mL})$ was prepared in methanol. Serial dilutions with methanol were made of each stock solution to prepare standard solutions of 50, 100, 250,500 and $1000 \mu \mathrm{g} / \mathrm{mL} ; 10 \mu \mathrm{L}$ of each standard solution was used for analysis and the construction of a standard calibration curve. The calibration curve of UA was linear and the regression equation of the peak area $(\mathrm{y})$ as a function of concentration $(\mathrm{x})$ was: $\mathrm{y}=473.2 \mathrm{x}+532.6(r=0.999)$. The concentration of UA in HCY2 was estimated to be $74.8 \%(\mathrm{w} / \mathrm{w})$. (HPLC-UV chromatography was provided as supplementary document) 


\subsection{Cell Culture}

$\mathrm{H} 9 \mathrm{c} 2$ cells, a permanent cell line derived from embryonic BD1X rat heart tissue, were purchased from American Type Culture Collection. The cells were cultured as monolayers in DMEM supplemented with $10 \%(\mathrm{v} / \mathrm{v})$ FBS. The medium contained $4.5 \mathrm{~g} / \mathrm{L}$ of glucose $(4.5 \mathrm{~g} / \mathrm{L})$ and glutamine (4.5 mM), supplemented with $\mathrm{NaHCO}_{3}(17 \mathrm{mM})$, penicillin $(100 \mathrm{IU} / \mathrm{mL})$ and streptomycin $(100 \mu \mathrm{g} / \mathrm{mL})$. All cells were grown under an atmosphere of $5 \% \mathrm{CO}_{2}$ in air $(\mathrm{v} / \mathrm{v})$ at $37{ }^{\circ} \mathrm{C}$. The medium was replaced every 2-3 days. A stock of cells was grown in a $75 \mathrm{~cm}^{2}$ culture flask and split before confluence at a subcultivation ratio of $1: 10$.

\subsection{Measurement of Mitochondrial Electron Transport in $\mathrm{H9c2}$ Cells}

$\mathrm{H} 9 \mathrm{c} 2$ cells were seeded in $75 \mathrm{~cm}^{2}$ culture flasks and allowed to grow to $70 \%-80 \%$ confluence prior to pre-incubation with the herbal extract. HCY2 (dissolved in DMSO) was added to the medium to achieve the desired final concentrations (DMSO $<0.2 \%, \mathrm{v} / \mathrm{v})$. After a 4-h incubation with HCY2 (10 or $25 \mu \mathrm{g} / \mathrm{mL}$ ), the HCY2-containing medium was aspirated and cells were trypsinized and permeabilized with digitonin $(50 \mu \mathrm{g} / \mathrm{mL})$. A mitochondria-rich fraction was isolated as described [26]. The measurement of electron transport in isolated mitochondria, which is based on the reduction of MTT, was performed using modified methods as described in [27]. An aliquot (40 $\mu \mathrm{L})$ of the mitochondria-rich fraction was mixed with $100 \mu \mathrm{L}$ each of $15 \mathrm{mM}$ pyruvate, $7.5 \mathrm{mM}$ malate and $0.42 \mathrm{mg} / \mathrm{mL}$ MTT. The reaction mixture was incubated at $37{ }^{\circ} \mathrm{C}$ for $10 \mathrm{~min}$ with gentle shaking. After the incubation, the reaction was terminated by the addition of $100 \mu \mathrm{L}$ lysis buffer $(10 \%$, w/v, sodium dodecyl sulfate and $45 \%$ dimethylformamide, adjusted to $\mathrm{pH} 4.7$ with glacial acetic acid). After standing for $5 \mathrm{~min}$, the absorbance at $570 \mathrm{~nm}$ of the reaction mixture was measured with a multi-titer plate reader (Bio-Rad, Hercules, CA, USA). Data were normalized to a mean control value of nonherbal extract pre-incubated samples and expressed as a percentage of control.

\subsection{Measurement of Mitochondrial ROS Production in H9c2 Cells}

$\mathrm{H} 9 \mathrm{c} 2$ cells were seeded in $75 \mathrm{~cm}^{2}$ culture flasks and allowed to grow to $70 \%-80 \%$ confluence prior to pre-incubation with the herbal extract. After a 4-h incubation with HCY2 (10 or $25 \mu \mathrm{g} / \mathrm{mL}$ ), a mitochondria-rich fraction was isolated as described above. An aliquot $(50 \mu \mathrm{L})$ of mitochondria-rich fraction (adjusted to $1 \mathrm{mg}$ protein/mL) was added to a well of a black multi-titer plate and incubated with $60 \mu \mathrm{L}$ of DCFDA (17.5 $\mu \mathrm{M}$ in incubation buffer) for $10 \mathrm{~min}$ at $37{ }^{\circ} \mathrm{C}$. After the incubation, an aliquot $(50 \mu \mathrm{L})$ of substrate mixture $(20 \mathrm{mM}$ pyruvate and $10 \mathrm{mM}$ malate) was added to each well. Fluorescence emission (excitation $485 \mathrm{~nm}$ and emission $535 \mathrm{~nm}$ ) was monitored every 5 min to until $30 \mathrm{~min}$ at $37^{\circ} \mathrm{C}$. ROS generation was calculated from fluorescence intensities after subtracting the fluorescence value of a blank sample containing only incubation buffer, substrate solution and DCFDA. The extent of ROS generation over the 30-min period was estimated by computing the area under the curve (AUC) of a graph plotting fluorescence intensity against time (0 to $30 \mathrm{~min}$ ). To investigate the effect of an antioxidant on HCY2-induced ROS production, cells were co-incubated with dimethylthiourea (DMTU $20 \mathrm{mM}$ ) and HCY2 $(25 \mu \mathrm{g} / \mathrm{mL})$ for $4 \mathrm{~h}$ and subjected to the 
measurement of mitochondrial ROS production as described above. The AUC of herbal extract preincubated samples were expressed as a percentage of the non-herbal extract pre-incubated controls.

\subsection{Measurement of Mitochondrial Membrane Potential in H9c2 Cells}

$\mathrm{H} 9 \mathrm{c} 2$ cells were seeded $\left(1.0 \times 10^{4}\right.$ cells $)$ on a 96-well black multi-titer plate with a clear bottom and were grown for $48 \mathrm{~h}$ before use. Cells were washed with phosphate buffered saline-A (PBS-A) twice and loaded with $100 \mu \mathrm{L}$ JC-1 (Sigma Chemical., St Louis, MO, USA) dye solution (20 $\mu \mathrm{M}$ in PBS-A) for 10 min under an atmosphere of $5 \% \mathrm{CO}_{2}$ in air $(\mathrm{v} / \mathrm{v})$ at $37{ }^{\circ} \mathrm{C}$. After staining with $\mathrm{JC}-1$, the cells were washed with PBS-A twice and incubated with HCY2 $(10$ or $25 \mu \mathrm{g} / \mathrm{mL})$ in the presence or absence of GDP $(500 \mu \mathrm{M})$. Carbonyl cyanide- $p$-trifluoromethoxyphenylhydrazone (FCCP, $100 \mu \mathrm{M})$, a chemical uncoupler, was used as a positive control in the measurement. The accumulation of JC-1 dye in mitochondria was quantified by red fluorescence intensity (excitation $527 \mathrm{~nm}$ and emission $590 \mathrm{~nm}$ ), which was monitored every $2 \mathrm{~min}$ to up to $40 \mathrm{~min}$ at $37^{\circ} \mathrm{C}$. The changes in mitochondrial membrane potential were expressed as the percentage of initial level of the corresponding control.

\subsection{Measurement of Mitochondrial Respiration in $\mathrm{H9c2}$ Cells}

Mitochondrial respiratory activity was measured polarographically with a Clark-type oxygen electrode (Hansatech Instruments, Norfolk, UK) as described [9]. H9c2 cells were seeded $\left(2.0 \times 10^{6}\right.$ cells) in $100 \mathrm{~mm}$ culture plate and pre-incubated with $\mathrm{HCY} 2(25 \mu \mathrm{g} / \mathrm{mL})$ for $4 \mathrm{~h}$ at $37{ }^{\circ} \mathrm{C}$. After the incubation, cells were collected by trypsinization and suspended in assay buffer $(120 \mathrm{mM} \mathrm{KCl}, 5 \mathrm{mM}$ $\mathrm{KH}_{2} \mathrm{PO}_{4}, 2 \mathrm{mM}$ EGTA, $10 \mathrm{mM}$ HEPES, $0.1 \mathrm{MgCl}_{2}, 0.5 \% \mathrm{BSA}, \mathrm{pH} 7.4$ ) and kept at $37{ }^{\circ} \mathrm{C}$. An aliquot $(1 \mathrm{~mL})$ of suspended cells $\left(1.5 \times 10^{6} \mathrm{cell} / \mathrm{s} / \mathrm{mL}\right)$ was placed in an air-tight liquid-phase oxygen electrode chamber. The system was maintained at $30{ }^{\circ} \mathrm{C}$ using a constant temperature water-jacketing system. Following equilibration, a non-ionic detergent, digitonin $(50 \mu \mathrm{g} / \mathrm{mL})$, was added and incubated for $3 \mathrm{~min}$ to permeabilize cell membranes. This was followed by the addition of pyruvate $(5 \mu \mathrm{M})$, malate $(2.5 \mu \mathrm{M})$ and ADP $(60 \mu \mathrm{M})$ to intiate mitochondrial state 3 respiration. Mitochondrial state 4 respiration was then induced by the addition of the specific complex V inhibitor, oligomycin $(1 \mathrm{mg} / \mathrm{mL})$. To investigate the role of UCPs in mitochondrial respiration, guanosine diphosphate (GDP, $500 \mu \mathrm{M}$ ) was added following the attainment of a steady state 4 respiration. For HCY2 pre-incubated cells, the rate of mitochondrial respiration was normalized to a mean control value from non-herbal extract pre-incubated cells and expressed as a percent of control.

\subsection{Measurement of Cellular GSH Levels in H9c2 Cells}

$\mathrm{H} 9 \mathrm{c} 2$ cells were seeded $\left(3.75 \times 10^{4}\right.$ cells/well $)$ in 12 -well culture plates. After stable attachment, cells were pre-incubated with HCY2 $(25 \mu \mathrm{g} / \mathrm{mL})$ in the absence or presence of DMTU $(20 \mathrm{mM})$ or bis-chloroethylnitrosourea (BCNU, $50 \mu \mathrm{M}$, a specific inhibitor of GR) at indicated time intervals at $37{ }^{\circ} \mathrm{C}$. Following the commencement of incubation, GSH levels were determined at increasing time intervals (4-24 h). Cellular GSH levels were determined enzymatically using DTNB [5,5'-dithiobis-(2nitrobenzoic acid)] and GR using a method modified from Griffith (1980) [28]. 


\subsection{Menadione-Induced Cytotoxicity in H9c2 Cells}

H9c2 cells used in the experiment were seeded at $3.75 \times 10^{4}$ cells in 12-well culture plate. After stable attachment, cells were pre-incubated with $\mathrm{HCY} 2$ for $4 \mathrm{~h}$ at $37{ }^{\circ} \mathrm{C}$. After the incubation with herbal extract, the herbal extract-containing medium was aspirated and the cells were incubated with menadione-containing medium $(12.5 \mu \mathrm{M})$ for another $4 \mathrm{~h}$ at $37^{\circ} \mathrm{C}$. For the non-menadione-challenged (non-Men) groups, the same volume of vehicle (i.e., ethanol) was added to the culture medium. After the menadione challenge, lactate dehydrogenase ( $\mathrm{LDH})$ activities in cell lysates and culture medium were measured in unchallenged and challenged cells, with or without herbal extract pre-incubation. The culture medium was collected and stored in a $1.5 \mathrm{~mL}$ micro-centrifuge tube at $4{ }^{\circ} \mathrm{C}$. Cells were then washed with PBS-A, and an aliquot $(300 \mu \mathrm{L})$ of lysis buffer [0.1\% (w/v) Triton X-100 in PBS] was added, and the mixture was incubated at $4{ }^{\circ} \mathrm{C}$ for $10 \mathrm{~min}$ prior to the LDH assay. LDH activity was measured as described by Li et al. [29]. The extent of LDH release was estimated as follows: [(LDH released into the medium in unchallenged or challenged cells) / (cellular LDH + LDH in the medium of unchallenged or challenged cells $) \times 100 \%$. The degree of protection against menadioneinduced cytotoxicity was estimated using the equation: [( $\left.\mathrm{LDH}_{\mathrm{Men} \text { Control }}-\mathrm{LDH}_{\text {non Men Herbal }}\right)^{-}$ $\left.\left(\mathrm{LDH}_{\text {Men Herbal }}-\mathrm{LDH}_{\text {non Men Herbal }}\right) /\left(\mathrm{LDH}_{\text {Men Control }}-\mathrm{LDH}_{\text {non Men Herbal }}\right) \times 100 \%\right]$.

\subsection{Statistical Analysis}

All data were expressed as mean \pm standard deviation (SD). Data were analyzed by one-way analysis of variance (one-way ANOVA) and intergroup differences were detected by the Scheffe method (single-step multiple comparison), with a value of $p<0.05$.

\section{Conclusions}

We can conclude that an ursolic acid-enriched active fraction derived from Herba Cynomorii protects against menadione cytotoxicity in $\mathrm{H} 9 \mathrm{c} 2$ cells. The cytoprotective effect may, at least in part, be attributed to an increase in mitochondrial uncoupling and glutathione redox cycling through the intermediacy of mitochondrial ROS production.

\section{Supplementary Materials}

Supplementary materials can be accessed at: http://www.mdpi.com/1420-3049/19/2/1576/s1.

\section{Acknowledgments}

We are thankful to David Godin (Department of Pharmacology and Therapeutics, The University of British Columbia, Vancouver, BC, Canada) for his useful comments and English-editing on the manuscript.

\section{Author Contributions}

Kam Ming Ko has been involved in designing the research and revising the paper; Jihang Chen has been involved in performing the research and analyzing data as well as drafting the paper; Hoi Shan Wong has been involved in discussing and interpreting the data 


\section{Conflicts of Interest}

The authors declare no conflict of interest.

\section{References}

1. Huss, J.M.; Kelly, D.P. Mitochondrial energy metabolism in heart failure: A question of balance. J. Clin. Invest. 2005, 115, 547-555.

2. Ballinger, S.W. Mitochondrial dysfunction in cardiovascular disease. Free Radic. Biol. Med. 2005, $38,1278-1295$.

3. Ko, K.M.; Mak, D.H.; Chiu, P.Y.; Poon, M.K. Pharmacological basis of 'Yang-invigoration' in Chinese medicine. Trends Pharmacol. Sci. 2004, 25, 3-6.

4. Meng, H.C.; Wang, S.; Li, Y.; Kuang, Y.Y.; Ma, C.M. Chemical constituents and pharmacologic actions of Cynomorium plants. Chin. J. Nat. Med. 2013, 11, 321-329.

5. Ma, C.; Nakamura, N.; Miyashiro, H.; Hattori, M.; Shimotohno, K. Inhibitory effects of constituents from Cynomorium songaricum and related triterpene derivatives on HIV-1 protease. Chem. Pharm. Bull. 1999, 47, 141-145.

6. Ma, C.M.; Wei, Y.; Wang, Z.G.; Hattori, M. Triterpenes from Cynomorium songaricium - analysis of $\mathrm{HCV}$ protease inhibitory activity, quantification, and content change under the influence of heating. J. Nat. Med. 2009, 63, 9-14.

7. Cui, Z.; Guo, Z.; Miao, J.; Wang, Z.; Li, Q.; Chai, X.; Li, M. The genus Cynomorium in China: An ethnopharmacological and phytochemical review. J. Ethnopharmacol. 2013, 147, 1-15.

8. Yu, F.R.; Liu, Y.; Cui, Y.Z.; Chan, E.Q.; Xie, M.R.; McGuire, P.P.; Yu, F.H. Effects of a flavonoid extract from Cynomorium songaricum on the swimming endurance of rats. Am. J. Chin. Med. 2010, 38, 65-73.

9. Chen, J.; Ko, K.M. Ursolic-acid-enriched herba cynomorii extract protects against oxidant injury in h9c2 cells and rat myocardium by increasing mitochondrial atp generation capacity and enhancing cellular glutathione redox cycling, possibly through mitochondrial uncoupling. Evid. Base Compl. Alternative Med. 2013, 2013, 924128:1-924128:14.

10. Ko, K.M.; Leon, T.Y.; Mak, D.H.; Chiu, P.Y.; Du, Y.; Poon, M.K. A characteristic pharmacological action of 'ang-invigorating' Chinese tonifying herbs: Enhancement of myocardial ATP-generation capacity. Phytomedicine 2006, 13, 636-642.

11. Chiu, P.Y.; Leung, H.Y.; Siu, A.H.; Chen, N.; Poon, M.K.; Ko, K.M. Long-term treatment with a Yang-invigorating Chinese herbal formula produces generalized tissue protection against oxidative damage in rats. Rejuv. Res. 2008, 11, 43-62.

12. Turrens, J.F. Mitochondrial formation of reactive oxygen species. J. Physiol. 2003, 552, 335-344.

13. Wong, H.S.; Chen, N.; Leong, P.K.; Ko, K.M. $\beta$-Sitosterol enhances cellular glutathione redox cycling by reactive oxygen species generated from mitochondrial respiration: Protection against oxidant injury in H9C2 cells and rat hearts. Phytother. Res. 2013, doi:10.1002/ptr.5087.

14. Hamanaka, R.B.; Chandel, N.S. Mitochondrial reactive oxygen species regulate cellular signaling and dictate biological outcomes. Trends Biochem. Sci. 2010, 35, 505-513.

15. Kowaltowski, A.J.; de Souza-Pinto, N.C.; Castilho, R.F.; Vercesi, A.E. Mitochondria and reactive oxygen species. Free Radic. Biol. Med. 2009, 47, 333-343.

16. Droge, W. Free radicals in the physiological control of cell function. Physiol. Rev. 2002, 82, 47-95. 
17. Sena, L.A.; Chandel, N.S. Physiological roles of mitochondrial reactive oxygen species. Mol. Cell 2012, 48, 158-167.

18. Echtay, K.S.; Roussel, D.; St-Pierre, J.; Jekabsons, M.B.; Cadenas, S.; Stuart, J.A.; Harper, J.A.; Roebuck, S.J.; Morrison, A.; Pickering, S.; et al. Superoxide activates mitochondrial uncoupling proteins. Nature 2002, 415, 96-99.

19. Bento, L.M.; Fagian, M.M.; Vercesi, A.E.; Gontijo, J.A. Effects of NH4Cl-induced systemic metabolic acidosis on kidney mitochondrial coupling and calcium transport in rats. Nephrol. Dial. Transplant. 2007, 22, 2817-2823.

20. Mari, M.; Morales, A.; Colell, A.; Garcia-Ruiz, C.; Fernandez-Checa, J.C. Mitochondrial glutathione, a key survival antioxidant. Antioxid. Redox Signal. 2009, 11, 2685-2700.

21. Kaminski, M.; Niemczyk, E.; Masaoka, M.; Karbowski, M.; Hallmann, A.; Kedzior, J.; Majczak, A.; Knap, D.; Nishizawa, Y.; Usukura, J.; et al. The switch mechanism of the cell death mode from apoptosis to necrosis in menadione-treated human osteosarcoma cell line 143B cells. Microsc. Res. Tech. 2004, 64, 255-258.

22. Chen, N.; Chiu, P.Y.; Leung, H.Y.; Ko, K.M. Cytochrome P-450-catalyzed reactive oxygen species production mediates the (-)schisandrin B-induced glutathione and heat shock responses in H9c2 cardiomyocytes. Indian J. Pharmacol. 2012, 44, 204-209.

23. Kossenjans, W.; Rymaszewski, Z.; Barankiewicz, J.; Bobst, A.; Ashraf, M. Menadione-induced oxidative stress in bovine heart microvascular endothelial cells. Microcirculation 1996, 3, 39-47.

24. McAmis, W.C.; Schaeffer, R.C.; Baynes, J.W.; Wolf, M.B. Menadione causes endothelial barrier failure by a direct effect on intracellular thiols, independent of reactive oxidant production. Biochim. Biophys. Acta 2003, 1641, 43-53.

25. Liobikas, J.; Majiene, D.; Trumbeckaite, S.; Kursvietiene, L.; Masteikova, R.; Kopustinskiene, D.M.; Savickas, A.; Bernatoniene, J. Uncoupling and antioxidant effects of ursolic acid in isolated rat heart mitochondria. J. Nat. Prod. 2011, 74, 1640-1644.

26. Wong, H.S.; Ko, K.M. Herba Cistanches stimulates cellular glutathione redox cycling by reactive oxygen species generated from mitochondrial respiration in $\mathrm{H} 9 \mathrm{c} 2$ cardiomyocytes. Pharm. Biol. 2013, 51, 64-73.

27. Cohen, G.; Farooqui, R.; Kesler, N. Parkinson's disease: A new link between monoamine oxidase (MAO) and mitochondrial electron flow. Proc. Natl. Acad. Sci. USA 1997, 94, 4890-4894.

28. Griffith, O.W. Determination of glutathione and glutathione disulfide using glutathione-Reductase and 2-vinylpyridine. Anal. Biochem. 1980, 106, 207-212.

29. Li, P.C.; Mak, D.H.; Poon, M.K.; Ip, S.P.; Ko, K.M. Myocardial protective effect of Sheng Mai San (SMS) and a lignan-enriched extract of Fructus Schisandrae, in vivo and ex vivo. Phytomedicine 1996, 3, 217-221.

Sample Availability: Samples of the Herbal Cynomorii extracts are available from the authors.

(C) 2014 by the authors; licensee MDPI, Basel, Switzerland. This article is an open access article distributed under the terms and conditions of the Creative Commons Attribution license (http://creativecommons.org/licenses/by/3.0/). 